\title{
Useful Application of Immunostaining to Malignant Pleural Effusion among Lao People in Vientiane Capital, Lao PDR
}

\author{
Soulideth Vilayvong ${ }^{1}$, Kiyomi Hando ${ }^{2}$, Masaki Sekine ${ }^{3}$, Thitsamay Luangxay ${ }^{1}$, \\ Phetsamone Arounlangsy ${ }^{4}$, Pheangvilay Xaysomphet ${ }^{4}$, Phetlammone Xayaphet ${ }^{4}$, \\ Hatthakone Thavisouk ${ }^{5}$, Yurie Soejima ${ }^{5}$, Masanobu Kitagawa ${ }^{1}$, Motoji Sawabe ${ }^{5 *}$
}

\begin{abstract}
Background: Pathology and laboratory medicine (PALM) services are limited in low-resource countries, such as Lao PDR. Patients with malignant pleural effusion (MPE) are not properly diagnosed and treated in these situations. The purpose of this study is to confirm the usefulness of immunocytochemistry in MPE to identify the histological type and probable primary site of cancer of MPE and to discuss its usefulness in low-resource countries, such as Laos. Methods: We retrospectively reviewed glass slides of pleural effusion sent to the Department of Pathology at the University of Health Sciences from the central hospitals for cytological screening from January 2012 to December 2016. The cytological review, cell transfer and immunocytochemical staining were performed at Tokyo Medical and Dental University. Among 81 cases of MPE from Laos, 66 cases of malignant tumors that contained enough tumor cells were included in this study, and the slides were screened with 14 primary antibodies to classify the histological type and identify the probable primary site of carcinoma. Results: Among the 66 cases, 34 cases (52\%) were of female patients, and 32 cases (48\%) were of male patients. The patients' ages ranged from 28 to 88 years with an average of 58 years. The immunocytochemical study identified 32 cases (49\%) of primary lung adenocarcinoma, two cases (3\%) of malignant mesothelioma, one case $(1.5 \%)$ of breast/gynecological carcinoma, one case (1.5\%) of T cell lymphoma, and one case $(1.5 \%)$ of B cell lymphoma. Twenty-nine cases $(43.5 \%)$ were classified as carcinoma not otherwise specified. Pulmonary small cell carcinoma/squamous cell carcinoma and metastatic colon, prostate, and liver carcinoma were not identified among the cases. Conclusions: Immunocytochemistry is a useful ancillary method in MPE diagnostics. This method could be applied in the pathological laboratories in low- or middle-resource countries, such as Laos.
\end{abstract}

Keywords: Malignant pleural effusion- immunocytochemistry- cytological cell transfer- health

Asian Pac J Cancer Prev, 20 (1), 243-248

\section{Introduction}

Malignant pleural effusion (MPE) is defined by the presence of malignant cells in the pleural effusion commonly seen in patients with advanced cancers. The incidence rate of MPE was estimated to be greater than 150,000 cases every year in the United States (Morgensztern et al., 2012), and 50,000 cases in the United Kingdom (Psallidas et al., 2016). Lung carcinoma is the most common origin of MPE (37.5\%), while $8 \%$ to $15 \%$ of patients with lung cancer had MPE (Morgensztern et al., 2012; Görgün et al., 2013). Breast cancer ranks as the second most common origin $(11.5 \%)$, followed by malignant lymphoma, including both Hodgkin's and non-Hodgkin's lymphoma (11.5\%) (Antunes et al., 2003). Tumors less commonly associated with MPE include gynecological and gastrointestinal carcinomas. MPE is an indicator of poor prognosis, and the estimated survival period ranges from 3 to 12 months (Antony et al., 2001). Information about the primary site, histological type, and occasionally the genotype is currently required to start the cancer treatment. The primary sites of cancer can be identified or speculated by various methods including serum tumor markers, medical imaging techniques such as computed tomography scan and magnetic resonance imaging (CT and MRI), and biopsies or fine needle aspiration cytology. As these examinations are scarce or unavailable in low-resource countries, the primary sites are frequently unidentified in cases with MPE. Cytological examination can usually determine the histological types of cancer such as adenocarcinoma, squamous cell carcinoma, small cell carcinoma, and malignant lymphoma; however, it frequently fails to determine the histological types. Cytological examination also cannot

${ }^{1}$ Department of Comprehensive Pathology, ${ }^{3}$ Department of Pathology, Medical Hospital, ${ }^{5}$ Department of Molecular Pathology, Graduate School of Medical and Dental Sciences, Tokyo Medical and Dental University, Tokyo, ${ }^{2}$ Department of Clinical Laboratory Sciences, Sanyo Women's College, Hiroshima, Japan, ${ }^{4}$ Department of Pathology, Faculty of Medicine, University of Health Care Sciences, Vientiane Capital, Lao PDR, Laos. *For Correspondence: m.sawabe.mp@tmd.ac.jp 
determine the primary site or the genotype of cancer. Immunocytochemistry (ICC) often provides useful information about the primary site, histological type, and the genotype of the cancer.

Lao PDR is a land-locked country situated in South East Asia, bordered by China, Myanmar, Vietnam, Cambodia, and Thailand. The population is 6.8 million (UNDP 2016). Lao PDR is classified as a low-income country with a poverty rate of $23 \%$ (The United Nations in Lao PDR, 2015). Health services in Laos are classified into four levels: health care centers, district hospitals, provincial hospitals, and central hospitals located in Vientiane Capital. Pathology and laboratory medicine (PALM) services are very limited in Laos and only available in three pathological laboratories in Vientiane Capital. Most of the pleural effusions were taken from patients admitted to one of the central hospitals and were sent for cytological screening to the pathological laboratory at the University of Health Sciences (UHS), the sole medical university in Laos. Due to poverty and low resources, only diagnostic thoracentesis is available in four central hospitals. Other pathological examinations of lung and pleural effusion such as bronchial lavage, pleural biopsy, medical thoracoscopy, bronchoscopy, and video-assisted thoracic surgery are not available in Laos. The cancer registry is under construction; likewise, no official or reliable data or research papers have yet been published on pleural effusion and lung cancer in Laos.

The purpose of the study is to confirm the usefulness of immunocytochemistry of MPE to examine the primary site, histological type, and the genotype of cancer of MPE and to discuss the usefulness of ICC of MPE in low resource countries, such as Laos.

\section{Materials and Methods}

\section{Sample collection}

We retrospectively reviewed glass slides of pleural effusion sent to the Department of Pathology of the Faculty of Medicine of UHS from the central hospitals for cytological screening from January 2012 to December 2016. The subjects were selected based on the cytological results and the volume of tumor cells on the glass slides; 81 cases that were suspected or positive for malignancy were first selected. These glass slides were brought to Japan, and the cytological review, cytological cell transfer and ICC were performed at Tokyo Medical and Dental University. This study was approved by the ethical committees in UHS (080/16) and in TMDU (M2017-051).

\section{Preliminary cytological screening}

Due to limited resources in Laos, all cytological smears were fixed with $10 \%$ formalin and stained with hematoxylin and eosin ( $\mathrm{H}$ and $\mathrm{E}$ staining). We removed the cover slips of all glass slides then restained with $\mathrm{H}$ and $\mathrm{E}$ to review the cytological features to check the histological types and volume of target tumor cells for cell transfer and immunocytochemistry. The cytological review was performed by three cytopathologists (S.V, K.H and M.S).

\section{Cell transfer technique (CTT)}

The CTT was conducted according to a previously published paper (Gong et al., 2005). After preliminary review, 66 of 81 cases which contained enough malignant cells were subjected for further immunocytochemical analysis. First, the slides with malignant cells were placed in xylene for one week to remove the cover slip. After the cover slip was removed, the slide was dipped in three changes of xylene in Coplin jars (10 dips per jar). A thin layer of Malinol (Muto Pure Chemical Co. Ltd., Tokyo, Japan), a mounting medium, was spread uniformly over the slide where the malignant cells were marked. The slide was left at room temperature for $30 \mathrm{~min}$, and then the slide was placed on a slide warmer (Sakura TissueTek, Slide Warmer, model: PS-53) at $60^{\circ} \mathrm{C}$ overnight. The next day, when the slide was completely dry and Malinol was rubbery and well attached to the cell, the slide was removed from slide warmer and left at room temperature for $30 \mathrm{~min}$. A slide marker was used to mark the malignant cells under the microscope. The slide was warmed in water at $60^{\circ} \mathrm{C}$ for one hour to soften the mounting medium. Then, Malinol containing malignant cells was peeled off with a carpel blade. The softened pliable medium was cut into small pieces that would contain malignant cells. The cut sections were pressed on the new slides in warm water $\left(60^{\circ} \mathrm{C}\right)$ for a few minutes, and the glass slides were placed on the cell warmer plate at $50^{\circ} \mathrm{C}$ overnight.

\section{Removal of $H$ and E staining}

After the slides were removed from the cell warmer plate, all slides were put on the plate at room temperature for $30 \mathrm{~min}$ and examined for the presence of malignant cells on each piece of the medium. The slides were submerged in three changes of xylene, $30 \mathrm{~min}$ for the first xylene change and $15 \mathrm{~min}$ for the second and third xylene change to remove Malinol. Then, the slides were dehydrated by submersion in two changes of $100 \%$ ethanol and one change of $70 \%$ ethanol (10 min per submersion). Then, the slides were rinsed in flowing water for $1 \mathrm{~min}$ and soaked in $1 \% \mathrm{HCl}$ in $70 \%$ alcohol for 4 hours or overnight (depending on the thickness of each cut section) to fade the color of hematoxylin. The slides were then rinsed with tap water and dehydrated with three changes of 100\% ethanol (10 min per submersion). After dehydration, the slides were placed in three changes of xylene for $10 \mathrm{~min}$ per each submersion and fixed with $95 \%$ ethanol for more than 30 min before immunocytochemical staining.

\section{Immunocytochemical staining}

We performed all the immunocytochemical procedures manually. First, the slides were rinsed in flowing tap water for a few minutes, and then the slides were dipped in distilled water for a few minutes. Heat-Induced Epitope Retrieval (HIER) was applied to all slides with Target Retrieval Solution, Citrate pH 6 (Code No. S.2639; Agilent Technologies Japan, Ltd., Tokyo, Japan) and incubation in boiling water at $98^{\circ} \mathrm{C}$ for $5 \mathrm{~min}$. The slides were cooled by incubation at room temperature $(10 \mathrm{~min})$ and placed in running tap water (10 min). After cooling, all slides were washed in distilled water by dipping gently 10 times. After rinsing, the slides were incubated in hydrogen peroxide 
3\% (Hydrogen peroxide 30\%, 081-04215, Wako Pure Chemical Industries, Ltd, Osaka, Japan) for $10 \mathrm{~min}$ to block endogenous peroxidase activity in the tissues, and then the slides were rinsed in running tap water for $2 \mathrm{~min}$. The slides were submerged in three changes of phosphate buffered saline $\mathrm{pH} 7$ and were soaked for 3 min each. The slides were drawn by liquid blocker pen (Liquid blocker supper pap pen, Daido Sangyo Co. Ltd. Tokyo, Japan). After drawing, the slides were dipped in 3 changes of phosphate buffered saline $\mathrm{pH} 7.4$ and were again soaked for 3 minutes each. The primary antibody was placed on every cut section and incubated at room temperature for 1 hour. Then, the slides were rinsed with 3 changes of phosphate buffered saline $\mathrm{pH} 7.4$ for 3 min each. The list of primary antibodies and their experimental conditions are shown in Table 1. The secondary antibody was placed on the cut sections and kept at room temperature for 30 min. Then, the slides were soaked with 3 changes of phosphate buffered saline $\mathrm{pH} 7.4$ for 3 minutes each. Chromogen substrate (AEC) (Histofine, simple stain AEC, 415182, Nichirei Biosciences INC, Tokyo, Japan) was used with all cut sections and kept at room temperature for $10 \mathrm{~min}$. Before rinsing AEC, we observed all slides under the microscope to confirm density of staining. Then, we rinsed the slides in running tap water for $5 \mathrm{~min}$. The slides were counterstained with Mayor Hematoxylin for $30 \mathrm{sec}$ to $1 \mathrm{~min}$. Before rinsing we observed the slides again. After that, we rinsed the slides in running tap water for $10 \mathrm{~min}$. Finally, the slides were mounted by Fluorescent Mounting Medium (Dako Fluorescent Mounting Medium, S 3023, Dako North America, Inc, California, USA).

\section{Diagnostic flow of immunocytochemistry}

The diagnostic flow of ICC is shown in Figure 1. At first, two suspected cases of malignant lymphoma were stained with CD3 and CD20 to distinguish the differentiation of lymphoma cells, such as $\mathrm{T}$ cell or $\mathrm{B}$ cell. TTF-1 was applied to all 66 cases to discriminate lung primary adenocarcinoma from other carcinomas.
Then, we applied Napsin A to identify primary lung adenocarcinoma among TTF-1 negative carcinoma cases. Among TTF-1 and Napsin A-double negative cases, ICC was performed with p40 for squamous cell carcinoma, Calretinin and D2-40 for malignant mesothelioma, CD56 and Chromogranin A for small cell lung carcinoma and neuroendocrine tumor, Hep Par-1 for hepatocellular carcinoma, and CDX2 for colorectal cancer. Female patients were screened with estrogen receptor (ER) and GCDFP-15 to identify gynecological and breast cancer, while male patients were screened with prostate-specific antigen (PSA) to determine cases of prostate carcinoma.

\section{Results}

\section{Cytological diagnosis}

Among 66 cases, 34 cases (52\%) were of female patients, and 32 cases $(48 \%)$ were of male patients. The age ranged from 28 to 88 years old with an average of 58 years. Forty patients $(61 \%)$ were residents of Vientiane Capital, and 26 patients (39\%) came from other provinces. All slides were prepared by Cytospin centrifuge preparation and stained with $H \& E$ staining. Preliminary review of the 66 cases revealed two cases of malignant lymphoma, and 64 cases of carcinoma not otherwise specified (carcinoma NOS). These 66 cases contained enough malignant cells for cell transfer and immunocytochemistry.

\section{Immunocytochemical results}

Figure 2 shows representative microscopic pictures of immunocytochemistry with selected primary antibodies. Among the 64 carcinoma cases, 25 cases were positive for TTF-1. Two cases of lymphoma were screened with CD20 and CD3: one case was CD20 positive and CD3 negative (B cell lymphoma), while another case was CD3 positive and CD20 negative (T cell lymphoma). Among the 39 TTF-1-negative carcinoma cases, seven cases were positive for Napsin A. A total of 32 cases (25 TTF-1 positive

Table 1. Primary Antibodies and Their Experimental Conditions

\begin{tabular}{|c|c|c|c|c|c|}
\hline Antibodies & Species & Type & Clone & Dilution & Manufacturer (Catalog number) \\
\hline Calretinin & rabbit & monoclonal & SP13 & Prediluted & Nichirei (413561) \\
\hline CD3 & mouse & monoclonal & F7.2.38 & $1: 50$ & DAKO (M7254) \\
\hline CD20 & mouse & monoclonal & L26 & $1: 100$ & DAKO (M0755) \\
\hline CD56 & mouse & monoclonal & CD564 & $1: 100$ & Novocastra (NCL-L-CD56-504) \\
\hline $\mathrm{CDX} 2$ & rabbit & monoclonal & EPR2764Y & Prediluted & Nichirei (418011) \\
\hline Chromogranin A & mouse & monoclonal & DAK-A3 & $1: 200$ & DAKO (M0869) \\
\hline D2-40 & mouse & monoclonal & D2-40 & $1: 50$ & DAKO (M3619) \\
\hline ER & rabbit & monoclonal & EPI & Prediluted & DAKO (IR084) \\
\hline GCDFP-15 & mouse & monoclonal & $23 \mathrm{~A} 3$ & $1: 50$ & Novocastra (PA0708) \\
\hline Hep Par-1 & mouse & monoclonal & OCH1E5 & $1: 200$ & DAKO (M7158) \\
\hline Napsin A & rabbit & polyclonal & n.a. & Prediluted & Nichirei (418061) \\
\hline p40 & rabbit & polyclonal & n.a. & Prediluted & Nichirei (418101) \\
\hline PSA & mouse & monoclonal & ER-PR8 & $1: 2000$ & DAKO (M0750) \\
\hline TTF-1 & mouse & monoclonal & $867 \mathrm{G} 311$ & $1: 200$ & DAKO (M3575) \\
\hline
\end{tabular}

CD, Cluster of differentiation; ER, Estrogen receptor; GCDFP-15, Gross Cystic Disease Fluid Protein-15; Hep Par-1, Hepatocyte Paraffin 1; HIER, Heat-induced epitope retrieval; n.a, not applied; PSA, Prostate-specific antigen; TTF-1, Thyroid transcription factor 1 


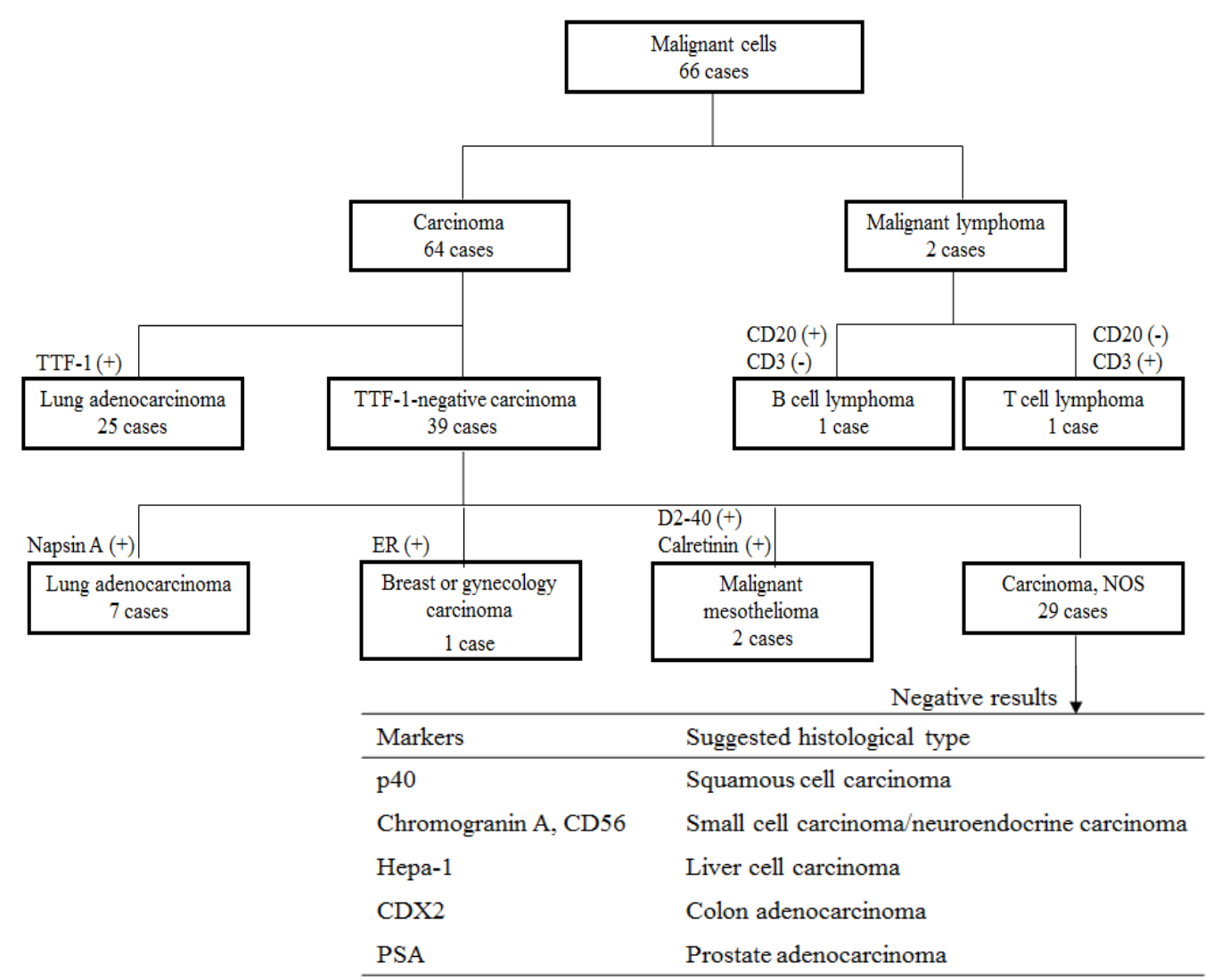

Figure 1. The Diagnostic Flow of Immunocytochemistry of Malignant Pleural Effusion

cases and seven TTF-1 negative, Napsin A-positive cases) were diagnosed as primary lung adenocarcinoma. Then, Calretinin and D2-40 immunostaining were performed; two cases were positive. Fifteen female cases among 32 non-lung carcinoma cases were screened with ER. Only one case was positive for ER; this case was negative for GCDFP-15. Twenty-nine cases of unclassified carcinomas were investigated by several primary antibodies: $\mathrm{p} 40$ for squamous cell carcinoma, chromogranin A and CD56 for small cell carcinoma and neuroendocrine tumor, Hep

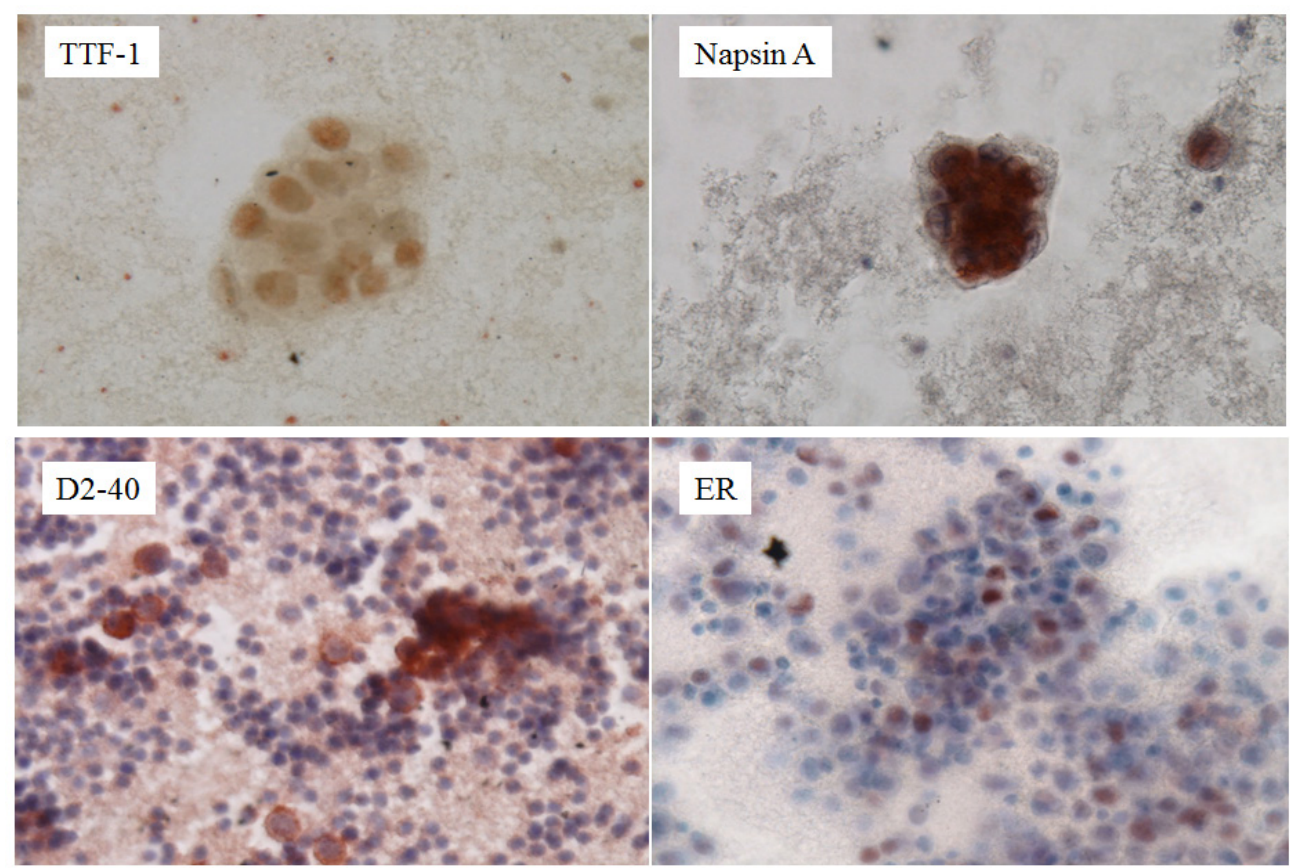

Figure 2. Representative Microscopic Pictures of Immunocytochemistry with Selected Pprimary Antibodies. (a), The nuclei of carcinoma cells are positive for TTF-1; (b), The cytoplasms of adenocarcinoma cells are positive for Napsin A; (c), The cytoplasms of tumor cells are positive for D2-40, suggesting malignant mesothelioma, while numerous inflammatory cells are negative; (d), Some carcinoma cells are positive for estrogen receptors in nuclei. TTF-1, Thyroid transcription factor 1. Immunostaining with DAB development and hematoxylin counterstaining. Magnification: x 200 
Par-1 for hepatocellular carcinoma, and CDX2 for colon cancer; all of them were negative. Among the 29 cases of unclassified carcinoma, male cases were checked by PSA to identify prostate carcinoma; all were negative.

Eventually, ICC with 14 markers identified 32 cases $(49 \%)$ of primary lung adenocarcinoma, two cases $(3 \%)$ of malignant mesothelioma, and one case each $(1.5 \%)$ of gynecological carcinoma, B cell lymphoma, and $\mathrm{T}$ cell lymphoma. The remaining 29 cases $(43.5 \%)$ were classified as carcinoma NOS.

\section{Discussion}

We performed an immunocytological study of MPE from 66 Lao patients. The lung seemed to be the primary site of origin in 32 cases (49\%). Two cases of malignant lymphoma were subtyped as B or T cell lymphoma. Two cases of malignant mesothelioma and one case of breast/ gynecological cancer were identified. In total, 37 cases $(56 \%)$ were immunocytologically characterized.

\section{TTF-1 and Napsin A}

TTF-1 and Napsin A are representative immunohistochemical markers of primary lung adenocarcinoma. TTF-1 is a transcription factor expressed in the nuclei of pulmonary alveolar lining cells and follicular cells of the thyroid. One study reported that $73 \%$ of primary lung adenocarcinomas were positive for TTF-1 (Liu and Farhood, 2004; Bishop et al., 2010). Napsin $\mathrm{A}$ is an aspartic proteinase, expressed in the cytoplasm of type II pneumocytes and alveolar macrophages. It is one of the highly sensitive markers of primary lung adenocarcinoma as well. Eighty-three percent of the primary lung adenocarcinomas were positive for Napsin A, while the squamous cell carcinomas were all negative (Fatima et al., 2011). Fatima et al., (2011) reported TTF-1/ Napsin A double immunostaining showed $74 \%$ sensitivity and $87 \%$ specificity in lung adenocarcinomas. This was also true in FNA cell block materials. In our study, 39\% $(25 / 64)$ of the carcinoma cases were stained with TTF1 , and 7 cases out of the 39 TTF-1-negative carcinoma cases were positive for Napsin A. We concluded a total of 32 cases $(48 \%)$ of MPE had the origin of primary lung adenocarcinoma.

p40

p40 has been widely used as a marker of primary lung squamous cell carcinoma. The sensitivity and specificity of p40 in squamous cell carcinoma was $76.9 \%$ and $93.3 \%$ with a lower sensitivity and higher specificity than those of p63 (Lilo et al., 2016). The combined immunostaining of TTF-1/Napsin A and p63(p40)/Cytokeratin 5 are helpful to distinguish between lung adenocarcinoma and squamous cell carcinoma, especially in the case of limited samples such as FNA cell blocks (Fatima et al., 2011). All cases among 29 unclassified carcinomas were negative for $\mathrm{p} 40$ in our study.

Calretinin and D2-40

The widely used markers of malignant mesothelioma are Calretinin and D2-40. A review of many articles showed that the sensitivity and specificity of D2-40 in malignant mesothelioma was 0.86 and 0.77 (He et al., 2017). Shield and Koivurinne, (2008) reported that $97 \%$ (33/34 cases) of cases of mesothelioma were positive for Calretinin, and the majority of the cases showed both cytoplasmic and nuclear staining, while only $3 \%(2 / 67)$ were positive for Calretinin in adenocarcinomas. Our study showed two out of the 39 TTF-1 negative cases were diffusely positive for both D20-40 and Calretinin.

\section{CD56 and Chromogranin A}

Identification of small cell lung carcinoma and neuroendocrine carcinoma is also important in MPE diagnostics. Kontogianni (2005) reported that CD56 was a useful marker in the diagnosis of small cell lung carcinoma, even in biopsies containing extensively collapsed carcinoma cells (nuclear smear) (Kontogianni et al., 2005). In our study, 29 cases of unclassified carcinoma were all negative for CD56 and Chromogranin A (another neuroendocrine marker).

\section{Estrogen receptor (ER)}

Estrogen receptor (ER) is a useful marker to distinguish between lung adenocarcinoma and metastatic carcinoma from the gynecological tract and breast (Kaufmann et al., 1998). The breast is one of the most common origins of MPE among female patients (Antunes et al., 2003). The individual sensitivities of ER and PR immunostaining in distinguishing breast cancer from other tumors were $72 \%$ and $52 \%$, respectively, while dual positive results increased the specificity of identifying a breast primary carcinoma to $84 \%$ (Lee et al., 2002). The GCDFP-15 is a marker of breast cancer, and only one case was ER-positive and GCDFP-15-negative among 15 female cases of TTF-1 negative carcinoma in our study.

\section{Hep Par-1}

As mentioned previously, the cancer registry system is still under construction in Laos. We could not estimate the exact number of cases of liver cancer in Laos. In Vientiane Capital, the service and condition of health facilities still cannot meet the population's demand. Therefore, middle-income and high-income Lao people usually seek better health facilities in Thailand or neighbor countries. Chaleunvong (2013), analyzing Lao patients who looked for cancer treatment in Thailand from Vientiane Capital, reported a high frequency of liver cancer patients $(16.1 \%$ in male and $7.2 \%$ in female patients) (Chaleunvong et al., 2013). Thus, some patients in our study might have had advanced liver cancer with MPE. However, the 29 cases of unclassified carcinomas were all negative for Hep Par-1, which is a useful marker for liver cancer (hepatocellular carcinoma). An ICC study of fine needle aspiration of a liver tumor showed Hep Par-1 positivity combined with Ber-EP4 negativity was exclusively observed in hepatocellular carcinoma (Onofre et al., 2007).

\section{Pathology and laboratory medicine (PALM) in low income countries}

The patient data storage and medical record system is not well organized in Lao hospitals. Thus, most of the patients' information is lost a few years after patients are 
discharged from the hospital. Clinical information and medical image films of the patients were also missing in this study. In addition, some data such as patient age is missing since the pathological laboratory of the University of Health Sciences is separately situated from the central hospitals, and patients' relatives must bring pathological samples to the university laboratory themselves. Difficulties often occur, such as poor fixation of the samples, lack of patients' clinical information, and loss of follow-up data of the patients. The importance of PALM services has recently been emphasized in the medical field. Wilson et al., (2018) reported that PALM services in low-income and middle-income countries should be cross-sectional and intersectional, providing safe, effective and equitable health care delivery and promoting population health and health care security. In reality, however, PALM services are severely inadequate, inequitable, and difficult to access among health services. In addition, sustainable development goals and universal health coverage cannot be achieved if PALM services have not been properly implemented in these countries. The most important barriers to accessing PALM services are as follows: insufficient human resources and workforce capacity, inadequate education and training of staff in pathological laboratories, inadequate infrastructure of laboratories, and insufficient quality, standards, and accreditation of the laboratory examination. In Laos, it is also necessary to begin increasing the number of healthcare workers, ameliorate task shifting and task sharing, improving the infrastructure of pathological laboratories, implementing integrated tiered systems of healthcare delivery and improving the accreditation of laboratories step by step. This point of view should be presented to Lao health sectors and related organizations.

In conclusion, immunocytochemistry is an effective and useful method in malignant pleural effusion diagnostics, providing the histological types and origins of tumors in at least half of cases, and thus facilitating clinical diagnosis and treatment choice. This method is applicable to PALM services even in low-income and middle-income countries.

\section{Financial support}

We received no financial support.

\section{Conflict of Interest}

We would like to disclose that there are no significant relations with financial interests or business purposes in this study.

\section{References}

Antony VB, Loddenkemper R, Astoul P, et al (2001). Management of malignant pleural effusions. Eur Respir J, 18, 402-19.

Antunes G, Neville E, Duffy J, et al (2003). BTS guidelines for the management of malignant pleural effusions. Thorax, 58, 29-38.

Bishop JA, Sharma R, Illei PB (2010). Napsin A and thyroid transcription factor-1 expression in carcinomas of the lung, breast, pancreas, colon, kidney, thyroid, and malignant mesothelioma. Hum Pathol, 41, 20-5.
Chaleunvong K, Kamsa-ard S, Suwanrungruang K, et al (2013). Retrospective appraisal of cancer patients from Vientiane Capital City, Lao People's Democratic Republic (PDR), seeking treatment in Thailand. Asian Pac J Cancer Prev, 14, 5435-40.

Fatima N, Cohen C, Lawson D, et al (2011). TTF-1 and Napsin A double stain: a useful marker for diagnosing lung adenocarcinoma on fine-needle aspiration cell blocks. Cancer Cytopathol, 119, 127-33.

Gong Y, Joseph T, Sneige N (2005). Validation of commonly used immunostains on cell-transferred cytologic specimens. Cancer, 105, 158-64.

Görgün D, Seçik F, Midilli K, et al (2013). Diagnostic and prognostic significance of survivin levels in malignant pleural effusion. Respir Med, 107, 1260-5.

He C, Wang B, Wan C, et al (2017). Diagnostic value of D2-40 immunostaining for malignant mesothelioma: a meta-analysis. Oncotarget, 8, 64407-16.

Kaufmann O, Kother S, Dietel M (1998). Use of antibodies against estrogen and progesterone receptors to identify metastatic breast and ovarian carcinomas by conventional immunohistochemical and tyramide signal amplification methods. Mod Pathol, 11, 357-63.

Kontogianni K, Nicholson AG, Butcher D, et al (2005). CD56: a useful tool for the diagnosis of small cell lung carcinomas on biopsies with extensive crush artefact. J Clin Pathol, $\mathbf{5 8}, 978-80$.

Lee BH, Hecht JL, Pinkus JL, et al (2002). WT1, Estrogen receptor, and progesterone receptor as markers for breast or ovarian primary sites in metastatic adenocarcinoma to body fluids. Am J Clin Pathol, 117, 745-50.

Lilo MT, Allison D, Wang Y, et al (2016). Expression of P40 and P63 in lung cancers using fine needle aspiration cases. Understanding clinical pitfalls and limitations. J Am Soc Cytopathol, 5, 123-32.

Liu J, Farhood A (2004). Immunostaining for thyroid transcription factor-1 on fine-needle aspiration specimens of lung tumors: a comparison of direct smears and cell block preparations. Cancer, 102, 109-14.

Morgensztern D, Waqar S, Subramanian J, et al (2012). Prognostic impact of malignant pleural effusion at presentation in patients with metastatic non-small-cell lung cancer. J Thorac Oncol, 7, 1485-9.

Onofre AS, Pomjanski N, Buckstegge B, et al (2007). Immunocytochemical diagnosis of hepatocellular carcinoma and identification of carcinomas of unknown primary metastatic to the liver on fine-needle aspiration cytologies. Cancer, 111, 259-68.

Psallidas I, Kalomenidis I, Porcel JM, et al (2016). Malignant pleural effusion: from bench to bedside. Eur Respir Rev, 25, 189-98.

Shield PW, Koivurinne K (2008). The value of calretinin and cytokeratin 5/6 as markers for mesothelioma in cell block preparations of serous effusions. Cytopathology, 19, 218-23.

United Nations in Lao PDR (2015). Country Analysis Report: Lao PDR. pages 1-147. web link: http://www.la.one.un.org/ media-center/publications/173-country-analysis-report-1 (accessed on August 1, 2018).

Wilson ML, Fleming KA, Kuti MA, et al (2018). Access to pathology and laboratory medicine services: a crucial gap. Lancet, 391, 1927-38.

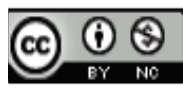

This work is licensed under a Creative Commons AttributionNon Commercial 4.0 International License. 